\title{
Mitral Valve Surgery: When Is It Appropriate?
}

\begin{abstract}
Mitral regurgitation (MR) is a frequent complication of end-stage cardiomyopathy. Historically, these patients were managed either medically or with mitral valve replacement, both associated with poor outcomes. We studied 150 patients with cardiomyopathy and severe MR who were managed with mitral reconstruction. One hundred fifty patients with 4+MR, left ventricular ejection fractions of 8\%-24\% (mean, 14\%), and New York Heart Association class III or IV symptoms were prospectively studied. All patients underwent mitral valve repair with an undersized, flexible annuloplasty ring. There was one intraoperative death and seven 30-day mortalities. Intraoperative echocardiography revealed no residual MR in the majority of patients and mild to trivial MR in seven patients. There were 27 late deaths; three of these patients had progression of the disease and underwent transplantation. The 1-, 2-, and 5-year actuarial survival rates are $82 \%, 71 \%$, and $57 \%$, respectively. New York Heart Association class has improved for all patients, from a preoperative mean of $3.2 \pm 0.2$ to $1.8 \pm 0.4$ postoperatively. At 24-month follow-up, all patients showed improvement in ejection fraction, cardiac output, and enddiastolic volumes, along with a reduction in the sphericity index and regurgitant volume. Mitral valve repair with an undersized, flexible annuloplasty ring is a safe and effective approach to correction of MR, even in cardiomyopathy patients. All observed changes contribute to reverse remodeling and the restoration of the normal left ventricular geometric relationship. Mitral reconstruction provides a new first-line management strategy for patients with MR and end-stage heart failure. (CHF. 2002;8:210-213) ๑2002 CHF, Inc.
\end{abstract}

Vinay Badhwar, MD; Steven F. Bolling, MD

From the Section of Cardiac Surgery, University of Michigan, Ann Arbor, MI

Address for correspondence:

Steven F. Bolling, MD, The University of Michigan

Hospitals, Section of Cardiac Surgery, 1500 E. Medical

Center Drive, 2120D Taubman Center, Box 0344, Ann

Arbor, MI 48109-0344

E-mail: sbolling@umich.edu

Manuscript received January 10, 2002;

accepted February 27, 2002
In our ever-aging population, advances in basic cardiac care that have extended our average life expectancy have also left more people living with chronic cardiac disease than ever before. In the United States alone, nearly 4.9 million people suffer from heart failure, yet of the 500,000 new patients diagnosed annually, fewer than 3000 are offered transplantation due to limitations of age, comorbid conditions, and donor availability. Therapeutic limitations have left a significant number of these patients and their physicians searching for alternate options. Despite improvements in the medical management of congestive heart failure (CHF), over $50 \%$ of patients continue to die within 3 years of presentation. 1,2 Those with CHF and mitral regurgitation (MR) have a life expectancy of less than 12 months. This clinical deficiency has led to the development of mitral reconstruction as a surgical alternative for the treatment of heart failure.

\section{Mitral Reconstruction}

Mitral regurgitation is a significant complication of end-stage cardiomyopathy and it affects almost all CHF patients as a preterminal event. Its presence is associated with progressive ventricular dilatation, an escalation of CHF symptomatology, and significant reductions in the long-term survival of these patients. ${ }^{3}$ The goal of surgical intervention, therefore, is to interrupt this cycle of heart failure through the restoration of normal cardiac physiology and ventricular mechanics.

Fundamental to the management of MR in heart failure is a firm understanding of the functional anatomy of the mitral valve. The mitral valve apparatus consists of the annulus, leaflets, chordae tendineae, and papillary muscles, as well as the entire left ventricle (LV). Thus, the maintenance of chordal, annular, and subvalvular continuity is essential for the preservation of mitral geometric relationships and overall ventricular function. As the ventricle fails, the progressive dilatation of the $\mathrm{LV}$ gives rise to MR, which begets more MR and further ventricular dilatation. Incomplete leaflet coaptation and regurgitation develops secondary to alterations in the annular-ventricular apparatus and ventricular geometry. ${ }^{4-5}$ These alterations include 
myocardial thinning and dilatation, blunting of the aortomitral angle, widening of the interpapillary distance, increased leaflet tethering, and decreased leaflet-closing forces. All of these combine to result in loss of the zone of coaptation (Figure 1). Thus, reconstructing this geometry serves not only to restore valvular competency but also to improve ventricular function. ${ }^{6-10}$

Historically, the surgical approach to MR was mitral valve replacement, yet little was understood of the interdependence of ventricular function and annulus-papillary muscle continuity. ${ }^{11}$ Consequently, patients with low ejection fractions who underwent mitral valve replacement with removal of the subvalvular apparatus had prohibitively high mortality rates. ${ }^{12}$ In an attempt to explain these outcomes, the concept of a beneficial "pop-off" effect of MR was conceived. This idea erroneously proposed that mitral incompetence provided low-pressure relief during systolic ejection from the failing ventricle, and that removal of this effect through mitral replacement was responsible for deterioration of ventricular function. Consequently, mitral valve surgery in patients with heart failure was discouraged. Renewed appreciation of the importance of subvalvular integrity in the preservation of post- operative LV function has led to new surgical techniques that have been applicable to patients with heart failure. ${ }^{13}$ Preservation of the mitral valve apparatus during mitral surgery has resulted in improved ventricular geometry, decreased wall stress, and improved systolic and diastolic function. 14 Thus, maintenance of chordal, annular, and subvalvular continuity is essential to surgically optimize mitral geometry and overall ventricular function. Moreover, with mitral repair, preservation of both leaflet integrity and the dynamic function of the mitral apparatus has clear long-term functional benefits.

In treating heart failure patients, the most significant determinant of leaflet coaptation and MR is the diameter of the mitral valve annulus. ${ }^{15}$ The left ventricular dimension is of less importance in functional $\mathrm{MR}$, as the length of the chordae and papillary muscles are similar in myopathic hearts regardless of whether MR is present. This may explain why undersizing and overcorrecting the mitral annulus during reconstruction not only serves to restore the zone of coaptation, but also permits its effective application to patients with diminished ejection fractions (Figure 2).

At the University of Michigan, 150 patients with end-stage cardiomyopathy and refractory, severe

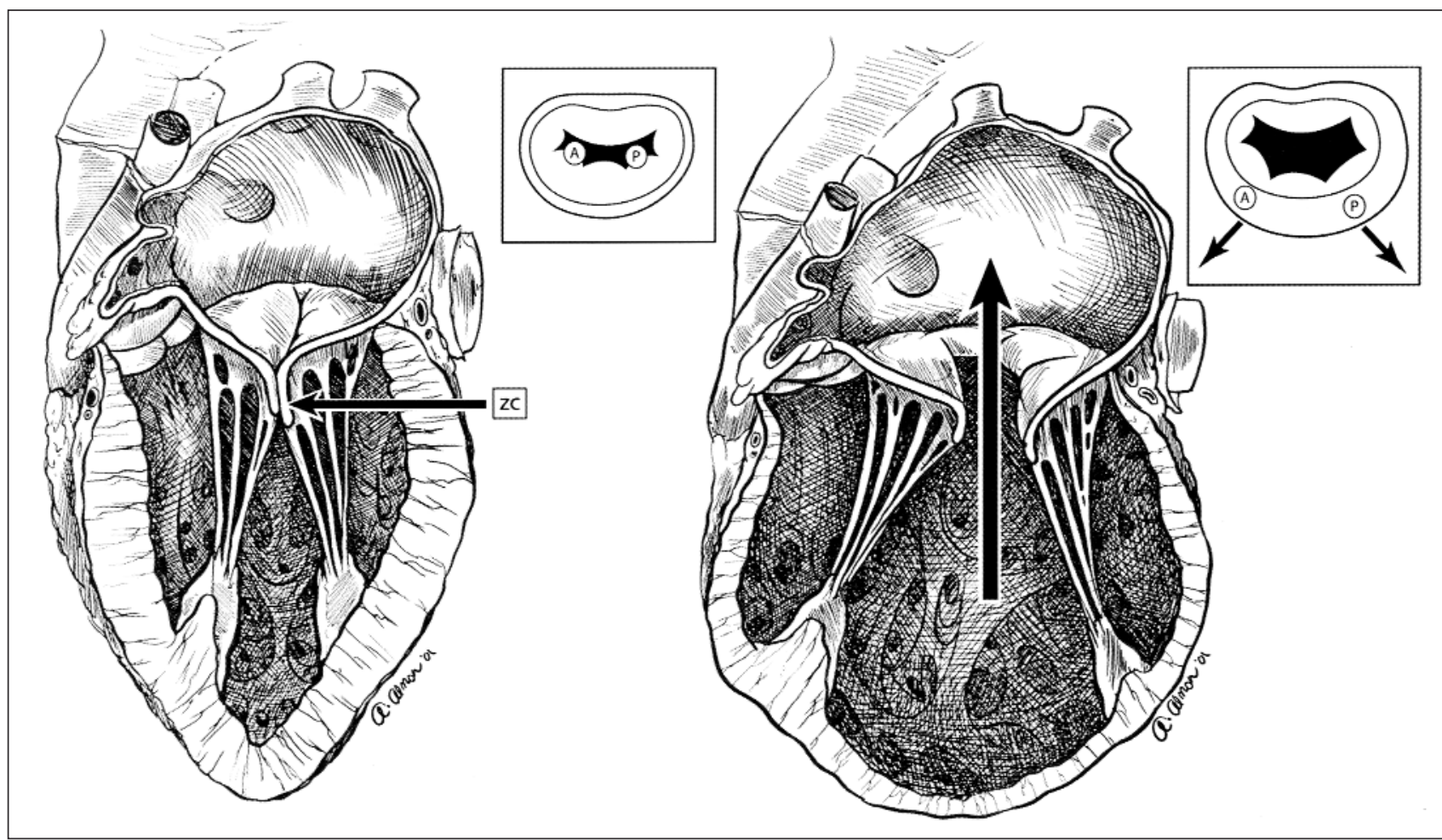

Figure 1. Morphologic changes in heart failure. Compared to the normal mitral apparatus and its effective zone of coaptation (ZC) on the left, note the changes that occur in heart failure, shown on the right. Alterations include myocardial thinning and dilatation, blunting of the aortomitral angle, widening of the interpapillary distance, increased leaflet tethering, and decreased leaflet-closing forces. This leads to altered force vectors on the papillary muscles (inset). All of these morphologic changes combine to result in loss of the zone of coaptation and central mitral regurgitation. 

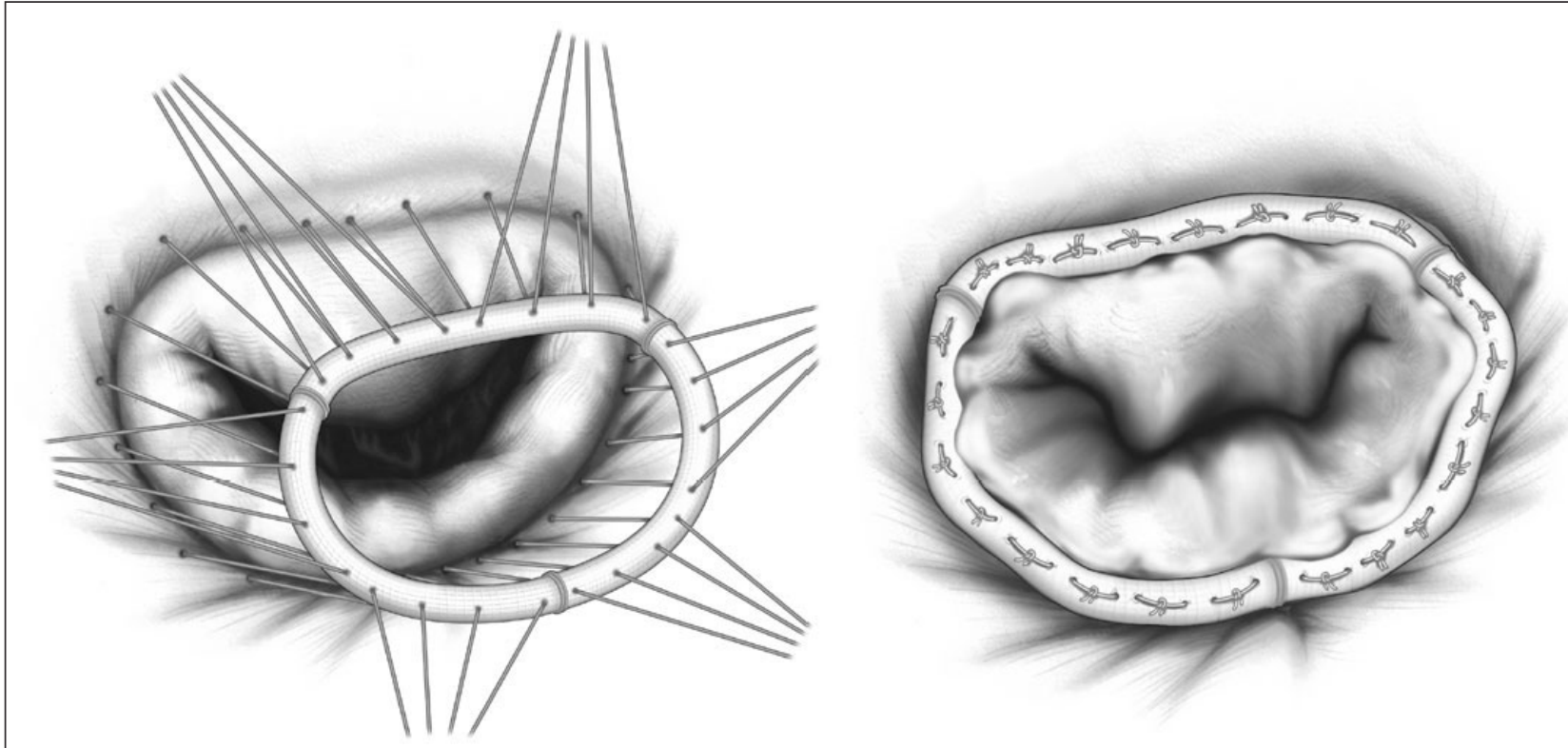

Figure 2. Geometric mitral reconstruction. Successful augmentation of the zone of coaptation and prevention of recurrent mitral regurgitation can be achieved by the placement of multiple annular sutures followed by an undersized, circumferential, flexible annuloplasty ring.

MR underwent mitral valve repair with an undersized, flexible annuloplasty ring (Figure 3). All patients were in New York Heart Association (NYHA) class III or IV heart failure despite receiving maximal medical therapy. Patients had severe LV systolic dysfunction, as defined by an ejection fraction of less than $25 \%$, with a mean of $14 \%$. On immediate postoperative echocardiograms, the mean transmitral gradient was $3 \pm 1 \mathrm{~mm} \mathrm{Hg}$ (range, 2-6 mm $\mathrm{Hg}$ ). The overall operative mortality was $5.3 \%$. There were eight 30-day mortalities: one intraoperative death due to right ventricular failure, one from a cerebrovascular accident, one from a pulmonary embolus, one from CHF, and four from multisystem organ failure. Five patients required intra-aortic balloon counterpulsation, and no patients required other forms of mechanical LV assistance. The duration of follow-up of these patients has been 1-80 months, with a mean of 43 months. There have been 27 late deaths: 12 from sudden ventricular arrhythmias, eight from progression of CHF but without MR, three related to complications from other operative procedures, three who progressed to transplantation, and one suicide. The one, two, and five year actuarial survival rates are $82 \%, 71 \%$, and $57 \%$, respectively.

At 24 months, all remaining patients were in NYHA class I or II, and had a mean ejection fraction of $26 \%$. NYHA symptoms were reduced from a preoperative mean of $3.2 \pm 0.2$ to $1.8 \pm 0.4$ postoperatively. These improvements paralleled subjective functional improvements reported by all patients. Echocardiographically, there were marked improvements in regurgitant fraction, end-diastolic volume, cardiac output, and sphericity index (Table). Although significant undersizing of the mitral annulus was employed to overcorrect for the zone of coaptation, no systolic anterior motion of the anterior leaflet or mitral stenosis was noted in these patients.

The technique of undersizing in mitral reconstruction probably avoids systolic anterior motion

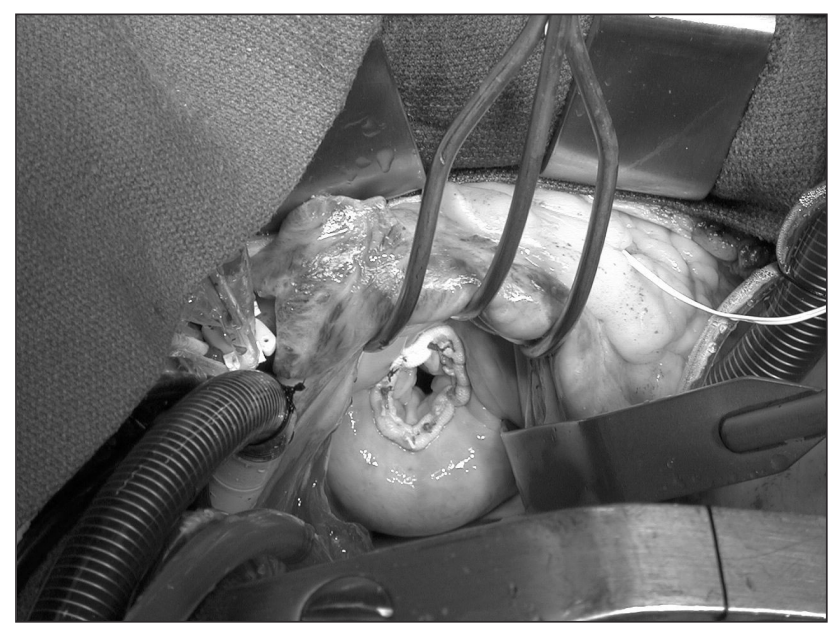

Figure 3. Completed mitral reconstruction. Surgical exposure is often enhanced by dissection of the interatrial groove prior to left atrial entry. The zone of coaptation in the dilated annulus of the myopathic patient can be readily restored without concern for systolic anterior motion and left ventricular outflow tract obstruction. 
Table. Matched Preoperative and Postoperative Data at 24 Months Following Mitral Reconstruction for Heart Failure

\begin{tabular}{|lccc|}
\hline $\begin{array}{c}\text { ECHO } \\
\text { PARAMETER }\end{array}$ & $\begin{array}{c}\text { POST } \\
\text { OPERATIVE }\end{array}$ & $\begin{array}{c}\text { OPERATIVE } \\
(24-\mathrm{MONTH})\end{array}$ & $\begin{array}{c}P \\
\text { VALUE }\end{array}$ \\
\hline $\begin{array}{c}\text { End-diastolic } \\
\text { volume (mL) }\end{array}$ & $281 \pm 86$ & $206 \pm 88$ & $<0.001$ \\
$\begin{array}{l}\text { Ejection } \\
\text { fraction (\%) }\end{array}$ & $16 \pm 5$ & $26 \pm 8$ & 0.008 \\
$\begin{array}{l}\text { Regurgitant } \\
\text { fraction (\%) }\end{array}$ & $70 \pm 12$ & $13 \pm 10$ & $<0.001$ \\
$\begin{array}{c}\text { Cardiac } \\
\text { output } \\
\text { (L/min) }\end{array}$ & $3.1 \pm 1.0$ & $5.2 \pm 0.8$ & 0.001 \\
$\begin{array}{c}\text { Sphericity } \\
\text { index (D/L) }\end{array}$ & $0.82 \pm 0.10$ & $0.74 \pm 0.07$ & 0.005 \\
\hline
\end{tabular}

in these myopathic patients through widening of the aortomitral angle in hearts with increased LV size. Furthermore, acute remodeling of the base of the heart with this reparative technique may also somewhat re-establish the normal geometry and ellipsoid shape of the LV. As evidenced by the decreased sphericity index and LV volumes seen in these patients, the geometric restoration of mitral reconstruction not only effectively corrects MR but also achieves surgical unloading of the ventricle.

\section{Comment}

Surgical therapies for the treatment of heart failure continue to evolve. Through improved preoperative management, intraoperative techniques, and postoperative care, the previous association of high mortality with surgical interventions for end-stage cardiomyopathy no longer applies. The combination of optimal medical management and mitral repair can provide cardiomyopathy patients with a long-term result that approaches that of transplantation, without the need for immunosuppression. Through the restoration of mitral competency and ventricular geometry, mitral reconstruction offers an appropriate new treatment strategy for the treatment of end-stage heart failure.

\section{REFERENCES}

1 Hunt SA. Current status of cardiac transplantation. JAMA. 1998;280(19):1692-1698.

2 Tavazzi L. Epidemiology of dilated cardiomyopathy: a still undetermined entity. Eur Heart J. 1997;18(1):4-6.

3 Blondheim DS, Jacobs LE, Kotler MN, et al. Dilated cardiomyopathy with mitral regurgitation: decreased survival despite a low frequency of left ventricular thrombus. $\mathrm{Am}$ Heart J. 1991;122(3 pt 1):763-771.

4 Boltwood CM, Tei C, Wong M, et al. Quantitative echocardiography of the mitral complex in dilated cardiomyopathy: the mechanism of functional mitral regurgitation. Circulation. 1983;68(3):498-508.

5 Kono T, Sabbah HN, Rosman H, et al. Left ventricular shape is the primary determinant of functional mitral regurgitation in heart failure. J Am Coll Cardiol. 1992;(7)20:1594-1598.

6 Bach DS, Bolling SF. Early improvement in congestive heart failure after correction of secondary mitral regurgitation in end-stage cardiomyopathy. Am Heart J. 1995;129:1165-1170.

7 Bolling SF, Deeb GM, Brunsting LA, et al. Early outcome of mitral valve reconstruction in patients with end-stage cardiomyopathy. J Thorac Cardiovasc Surg. 1995;109(4):676-683.

8 Bach DS, Bolling SF. Improvement following correction of secondary mitral regurgitation in end-stage cardiomyopathy with mitral annuloplasty. Am J Cardiol. 1996;78:966-969.

9 Bolling SF, Pagani FD, Deeb GM, et al. Intermediate-term outcome of mitral reconstruction in cardiomyopathy. J Thorac Cardiovasc Surg. 1998;115:381-388.

10 Chen FY, Adams DH, Aranki SF, et al. Mitral valve repair in cardiomyopathy. Circulation. 1998;98:II124-II127.

11 Pitarys CJ II, Forman MB, Panayiotou H, et al. Long-term effects of excision of the mitral apparatus on global and regional ventricular function in humans. J Am Coll Cardiol. 1990;15(3):557-563.

12 Phillips HR, Levine FH, Carter JE, et al. Mitral valve replacement for isolated mitral regurgitation: analysis of clinical course and late postoperative left ventricular ejection fraction. Am J Cardiol. 1981;48(4):647-654.

13 David TE, Uden DE, Strauss HD. The importance of the mitral apparatus in left ventricular function after correction of mitral regurgitation. Circulation. 1983;68(3 pt 2):II76-1182.

14 Sarris GE, Cahill PD, Hansen DE, et al. Restoration of left ventricular systolic performance after reattachment of the mitral chordae tendineae. The importance of valvular-ventricular interaction. J Thorac Cadiovasc Surg. 1988;95(6):969-979.

15 Rosario LB, Stevenson LW, Solomon SD, et al. The mechanism of decrease in dynamic mitral regurgitation during heart failure treatment: importance of reduction in the regurgitant orifice size. J Am Coll Cardiol. 1998;32:1819-1824. 